Schweizerisches Jahrbuch für Entwicklungspolitik

26-2 | 2007

Entwicklungsfinanzierung durch Mobilisierung lokaler Ressourcen

\title{
Kreditmangel oder Gewinnausfall? \\ Familienwirtschaft in Vietnam
}

\section{Christophe Gironde}

\section{(2) OpenEdition}

\section{Journals}

Electronic version

URL: http://journals.openedition.org/sjep/402

DOI: $10.4000 /$ sjep.402

ISSN: 1663-9677

\section{Publisher}

Institut de hautes études internationales et du développement

\section{Printed version}

Date of publication: 1 novembre 2007

Number of pages: 165-181

ISBN: 978-2-88247-069-0

ISSN: 1660-5926

Electronic reference

Christophe Gironde, « Kreditmangel oder Gewinnausfall? Familienwirtschaft in Vietnam »,

Schweizerisches Jahrbuch für Entwicklungspolitik [Online], 26-2 | 2007, Online erschienen am: 03 Juni

2010, abgerufen am 08 September 2020. URL : http://journals.openedition.org/sjep/402 ; DOI :

https://doi.org/10.4000/sjep.402 


\title{
Kreditmangel oder Gewinnausfall? Familienwirtschaft in Vietnam
}

\author{
Christophe Gironde*
}

\section{Einführung}

Die Behauptung, den Bauern fehle es an Fremdkapital, ist nicht neu. Im Falle Vietnams aber ist sie seit der Rehabilitierung der Familienwirtschaft unvermindert aktuell und eines der Hauptargumente für die Begründung, weshalb ihrer Entwicklung Grenzen gesetzt seien. Als Antwort darauf wurden zahlreiche Mikrokreditprojekte ins Leben gerufen, deren Bilanz gerade in Vietnam häufig positiv bewertet wird ${ }^{1}$. Fehlt es den vietnamesischen Bauern tatsächlich an Fremdkapital? Dieser zentralen Frage soll in diesem Beitrag nachgegangen werden. Ausgangspunkt der Überlegungen bilden die Ergebnisse aus der Feldforschung, die seit Mitte der 1990er-Jahre im Delta des Roten Flusses und insbesondere in den Landkreisen Khoai Châu und Van Giang (Provinz Hung Yên) durchgeführt wird, einer Region, die sich durch eine besonders dynamische Entwicklung des Agrarsektors und eine ausgeprägte Diversifizierung der Wirtschaftstätigkeit auszeichnet ${ }^{2}$.

Die Auflösung der landwirtschaftlichen Kooperativen im Laufe der 1980erJahre fand vor dem Hintergrund eines Mangels an Lebensmitteln, Hilfsstoffen, Konsumgütern und Fremdkapital statt. Die Rehabilitation der Familienbetriebe als kleinster Einheit der Agrarwirtschaft führte sehr bald $\mathrm{zu}$ einer enormen Nachfrage nach Kapital, die die Kapazitäten des formellen Finanzsektors Ende der 1980er- und Anfang der 1990er-Jahre überstieg ${ }^{3}$ Der Mangel an Fremdkapital beherrschte zu jener Zeit die Debatte über die Entwicklung des Agrarsektors, deren Hauptaugenmerk der Steigerung der Produktionskapazitäten galt, lautete doch das erklärte Ziel, mit der Verschwendung der planwirtschaftlichen Kollektive zu brechen, die Ernährungssicherheit der Bevölkerung zu gewährleisten und Überschüsse für den Export zu produzieren. Im Laufe der 1990er-Jahre dann weitete sich das Kreditangebot in den ländlichen Regionen Vietnams beträchtlich aus und ermöglichte tief greifende Umstrukturierungen auf der Ebene der einzelnen Familienbetriebe. Nun spielen Kredite unbestrittenermassen eine

* Lehrbeauftragter am Institut universitaire d'études du développement (IUED), Genf.

1 M. Leflart, Intérêt et apport du micro-crédit, Hanoi, Forum économique et financier franco-vietnamien - Le financement du développement, 19.-20. Januar 2005, S. 2.

2 C. Gironde, Réhabilitation et transformations de l'économie familiale au Nord-Vietnam. Systèmes d'activités villageois et réseaux de relations dans le delta du fleuve Rouge, thèse de doctorat, Genève, Institut universitaire d'études du développement, 2001, 406 S.

3 A.-C. Creusot, Quynh Tran Thi Thanh und Luong Quoc Tuân, „La microfinance a-t-elle encore une place lorsque l'offre de crédit s'étend ?", La microfinance en Asie. Entre traditions et innovations, Pondichéry, Institut français de Pondichéry; Paris, Institut de recherche pour le développement ; Karthala, 2005, S. 64-82. 
wichtige Rolle. Dennoch scheinen sie nicht zwangsläufig eine Vorbedingung für solche Umstellungen zu sein: In der Tat ist die Familienwirtschaft zu ausserordentlichen Entwicklungen fähig, ohne dass im Vorfeld bedeutende Mengen an Ressourcen angehäuft wurden. Desgleichen vermag auch die zunehmende Verfügbarkeit von Fremdkapital gewisse Schranken nicht aufzuheben, die der Familienwirtschaft gesetzt sind und die sich hauptsächlich in der geringen Rentabilität der Produktionstätigkeit bzw. in einem Mangel an Profit äussern.

\section{Der Mangel an Fremdkapital}

\section{Die Kreditproblematik im Übergang zur Marktwirtschaft}

\section{$\square$ Rückblick auf die Ära der Planwirtschaft}

Bis in die zweite Hälfte der 1980er-Jahre bestand das vietnamesische Bankensystem aus mehreren sektorspezifischen Banken, die von der Zentralbank (der vietnamesischen Staatsbank) und dem Finanzministerium kontrolliert wurden. Diese beiden Instanzen waren weitgehend an die Weisungen und Entscheide des staatlichen Planungsausschusses gebunden ${ }^{4}$. Die Defizite der Staatsbetriebe wurden durch den Staatshaushalt gedeckt, der seinerseits dank der Notenpresse, Krediten der Zentralbank sowie Hilfszahlungen der Mitgliedsländer des Rates für gegenseitige Wirtschaftshilfe buchhalterisch ,ausgeglichen“ wurde 5 .

Die Finanzierung der Agrarwirtschaft (siehe unten Schema 1) war offiziell und nahezu ausschliesslich ${ }^{6}$ durch staatliche Strukturen sichergestellt: Die Finanzmittel der Kooperativen und staatlichen Betriebe stammten von der für Agrarkredite zuständigen Abteilung der Zentralbank. Die Staatsbetriebe belieferten die Produzentengruppen direkt mit Hilfsstoffen und Materialien und wurden dafür in Form von Reis bezahlt. Ab 1956 entstanden im Norden, ab 1983 auch im Süden Kreditgenossenschaften. Ihre Aufgabe war es, kleine Spareinlagen entgegenzunehmen und den Familien Kredite zu gewähren. Zu Beginn der 1960er-Jahre zählte das Land rund 5500 solcher Kreditgenossenschaften. Anfang der 1980er-Jahre allerdings stieg deren Zahl mit der Gründung von mehr als 2000 neuen Kreditgenossenschaften sprunghaft an. Diese Spar- und Leihkassen standen theoretisch unter der Kontrolle der Zentralbank. In der Praxis hingegen wurden sie von lokalen Ausschüssen geführt.

\section{$\square$ Reformen und Finanzkrise}

1986 proklamierte Vietnam eine wirtschaftliche Neuausrichtung: Unter dem Namen Dôi Moi („Erneuerung“) wurden institutionelle und makroökonomische Reformen in die Wege geleitet, die den Finanzierungskontext grundlegend umwälzten. Das erklärte Ziel lautete nunmehr, die Staatsbetriebe in Beteiligungs-

4 C. Feuché, „Croissance, Etat et marché dans le Vietnam du Dôi Moi“, in S. Dovert und B. de Tréglodé (dir.), Vietnam contemporain, Paris, Les Indes Savantes, 2004, S. 246.

5 E. Paquet, Le système économique vietnamien 1979-2000. De la réforme à la transformation, thèse de doctorat, Paris, Université Paris VII - Denis Diderot, Juni 2003, S. 187.

6 Der Umfang der Aktivitäten, die ausserhalb der Kooperativen bzw. auf private Initiative der Haushalte stattfanden und aus abgezweigten Mitteln der Kooperativen finanziert wurden, ist nicht zu unterschätzen. 
gesellschaften umzuwandeln, ausländische Investitionen anzulocken und die Sparguthaben der Bürgerinnen und Bürger zu mobilisieren. Als Erstes begann der Staat, seine Ressourcen rationeller einzusetzen: Unrentablen Staatsbetrieben drohte die Schliessung, die übrigen mussten ihren Finanzbedarf künftig statt über direkte Subventionszahlungen durch Kredite der staatlichen Bank decken.

Die Subventionen für die landwirtschaftlichen Kooperativen wurden eingestellt. Auch sie mussten zur Deckung ihres Mittelbedarfs Darlehen bei den örtlichen Kreditgenossenschaften aufnehmen. In dieser Übergangsphase liehen sich zahlreiche Betriebe trotz rückläufiger Produktionstätigkeit weiterhin Geld aus. Die Tatsache, dass die Kredite nicht getilgt wurden, aber auch Betrug und Unterschlagung leisteten der drohenden Krise Vorschub. Diese brach Ende der 1980er-Jahre in voller Wucht aus, als die Zentralbank die automatische Refinanzierung der Kreditgenossenschaften einstellte ${ }^{7}$. Ende 1990 hatten von den ursprünglich 7180 Kreditgenossenschaften des Landes nur gerade 160 überlebt. Die Einlagen der Bürgerinnen und Bürger waren verloren, das Vertrauen der Bevölkerung zutiefst erschüttert ${ }^{8}$.

In der Zwischenzeit, genauer 1988, war die staatliche Agrarentwicklungsbank (VBARD) gegründet worden. Diese öffentliche Geschäftsbank sollte den Agrarsektor - und mithin die Familienbetriebe als kleinste Produktionseinheiten - mit den nötigen Finanzmitteln versorgen. In einer ersten Phase finanzierte die VBARD vor allem die Staatsbetriebe: Zu Beginn der 1990er-Jahre entfielen $80 \%$ aller gewährten Kredite auf staatliche Unternehmen ${ }^{9}$. Demgegenüber stellten die Kredite an Familienbetriebe Ende 1991 nur gerade $10 \%$ des gesamten Kreditvolumens ${ }^{10}$. Die Reform des Bankensektors vermochte mit den institutionellen Reformen nicht Schritt zu halten ${ }^{11}$ : Just zu der Zeit, als die Staatsunternehmen investieren mussten, um ihre Rentabilität $\mathrm{zu}$ sichern und einer Schliessung zu entgehen, wurde immer mehr Budgetdisziplin gefordert. Die schrittweise Anerkennung des Privatsektors schaffte enorme Anreize zur Unternehmensgründung, gleichzeitig aber fehlte es an Banken, die diesen im Entstehen begriffenen Sektor mit Geld versorgen konnten. Die Bauern waren gezwungen, ihre Produktionstätigkeit aus eigener Kraft zu finanzieren. Der Bedarf an Finanzmitteln wuchs rapide an, denn die Diversifizierung des Getreideanbaus und der Viehzucht und der Aufbau nichtlandwirtschaftlicher Tätigkeiten waren kapitalintensiv.

7 A. Wolz, The Transformation of Rural Finance Systems in Vietnam, Discussion Paper, Nr. 60, Research Centre for International Agrarian and Economic Development, Heidelberg, Universität Heidelberg, 1997, S. 6.

8 C. Poursat, „Le crédit aux paysans“, in Association d'amitié franco-vietnamienne (dir.), L'agriculture et la paysannerie vietnamiennes, Paris, L'Harmattan, 2000, S. 66.

9 A.-C. Creusot, Le paysage du financement du monde rural au Vietnam, Bulletins d'information du mardi (Pôle microfinancement du Centre de coopération internationale en recherche agronomique pour le développement [CIRAD] et du Groupe de recherche et d'échanges technologiques [GRET]), Nr. 16, 14. Mai 2002, S. 1, <http://microfinancement.cirad.fr/fr/news/bim/Bim-2002/BIM-14-0502.pdf>.

10 A. Wolz, op. cit., S. 7.

11 E. Paquet, op. cit., S. 208. 


\section{Schema 1: Finanzierung des Agrarsektors in der Ära der Planwirtschaft}

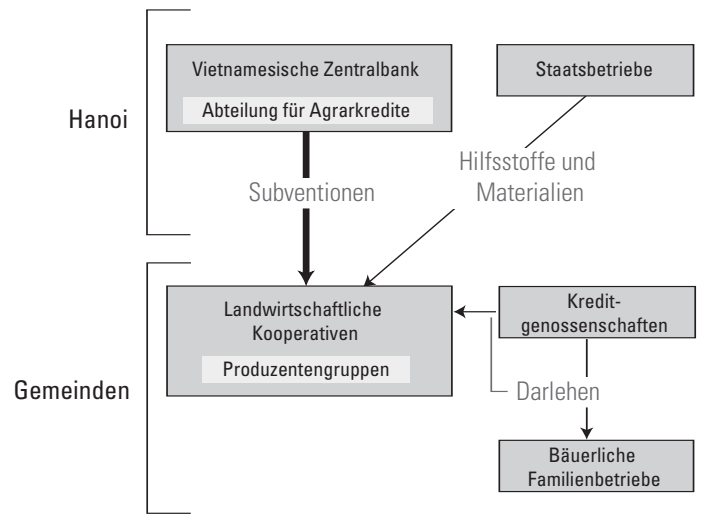

\section{Die These vom Mangel an Fremdkapital}

Im Zuge der Reformen wuchs der Kapitalbedarf in sämtlichen Bereichen der vietnamesischen Wirtschaft rapide an, sei es in den zur Modernisierung gezwungenen Staatsbetrieben, in den unlängst anerkannten Privatunternehmen oder in der rehabilitierten Familienwirtschaft. Das Wirtschaftswachstum sollte zudem eine zahlungsfähige Nachfrage hervorbringen, die den Bedarf an Konsumkrediten in die Höhe schnellen liess.

Sehr rasch wurden die Investitionskapazität und der Mangel an Fremdkapital zu Schlüsselfragen im Hinblick auf die Möglichkeiten und Grenzen von Dôi Moi : Die Fähigkeit, innert kürzester Zeit Kapital anzuhäufen, galt als entscheidend für den Erfolg bzw. den Misserfolg der im Privatsektor entstehenden Unternehmen $^{12}$. Dem Mangel an Kapital sei es zu verdanken, dass manche registrierte Privatunternehmen letztlich nur auf dem Papier bestünden und keinerlei Unternehmenstätigkeit ausübten ${ }^{13}$.

Die ländlichen Gebiete hätten unter dem mangelhaften Kreditangebot seitens der Finanzinstitute zu leiden, sodass für die Bauern der Zugang zu Krediten wichtiger werde als deren Kosten ${ }^{14}$. Dadurch werde die Entwicklung der ländlichen Gebiete im Allgemeinen und der Familienbetriebe und Unternehmen des Agrarsektors im Besonderen erheblich behindert ${ }^{15}$. Dieses Defizit zeige, dass im

12 P. Ronnas und B. Ramamurthy, Entrepreneurship in Vietnam: Transformation and Dynamics, Copenhagen, Nordic Institute of Asian Studies; Singapore, Institute of Southeast Asian Studies, 2001, S. 204. C. Feuché, op. cit., S. 241.

13 Hoang Kim Giao und Hoang Vu Cuong, ,Vietnam's Private Economy in the Process of Renovation“, in I. Norlund, C. L. Gates und Vu Cao Dam (eds.), Vietnam in a Changing World, Richmond, UK, Curzon Press, 1995, S. 151.

14 C. Poursat, op. cit., S. 73. A. Johnson, Microfinance in Vietnam: A Collaborative Study Based upon the Experiences of NGOs, UN Agencies and Bilateral Donors, Hanoi, United Nations Development Programme (UNDP); Consultative Group to Assist the Poor (CGAP), 1996.

15 Bui Van Hung, „Rural Diversification: An Essential Path to Sustainable Development for Vietnam“, in M. Beresford und Tran Ngoc Angie (eds.), Reaching for the Dream: Challenges of Sustainable Development in Vietnam, Copenhagen, Nordic Institute of Asian Studies ; Singapore, Institute of Southeast Asian Studies, 2004, S. 198. 
vorgelagerten Bereich die Mobilisierung von Spareinlagen unzureichend sei (die Einlagen verzeichneten zwar ein Wachstum, aber es handelte sich dabei hauptsächlich um kurzfristige Gelder ${ }^{16}$ ), während im nachgelagerten Bereich die Marktmechanismen den Zugang zu Finanzmitteln nicht zu gewährleisten vermöchten ${ }^{17}$. Zahlreiche Untersuchungen auf Betriebsebene wiesen eine Korrelation zwischen Kapitalisierung und Rentabilität der Anbausysteme nach: je kapitalintensiver die Produktionssysteme, desto grösser der Profit ${ }^{18}$. Um es mit den Worten von N. Perrin zu sagen: Die Finanzierungskapazitäten wirkten sich diskriminierend aus ${ }^{19}$. Nicht zuletzt gilt der Zugang zu Krediten als elementare Voraussetzung im Kampf gegen die Armut. Der diagnostizierte Mangel an Kreditmöglichkeiten führte denn auch in den 1990er-Jahren zur Entstehung zahlreicher Mikrofinanzprojekte, die sich im Wesentlichen auf das Mikrokreditgeschäft konzentrierten ${ }^{20}$.

Allerdings gibt es auch Analysen, die zu anderen Ergebnissen kommen. Diese Stimmen sind jedoch gegenüber denjenigen der Entwicklungshilfeorganisationen und ihrer vietnamesischen Partnerinstitutionen in der Minderheit. Laut Hoang Kim Giao und Hoang Vu Cuong schöpfen selbst Unternehmen, die Zugang zu Krediten haben, das Investitionspotenzial nicht aus. Der Grund dafür seien die für eine Expansion der Unternehmenstätigkeit zu unsicheren Gewinnaussichten $^{21}$. Tran Thi Quê und Hong Phan stellen ebenfalls fest, die Bauern seien angesichts der ungewissen Einkommensaussichten wenig geneigt, Fremdkapital aufzunehmen ${ }^{22}$. Laut Abrami und Henaff wäre es ein Fehler, zu glauben, Privatunternehmen seien nur deshalb klein, weil ihnen das für eine Expansion benötigte Kapital fehle. Vielmehr strebten sie insbesondere in ländlichen Regionen danach, durch eine Begrenzung ihres Geschäftsvolumens eine Besteuerung und bürokratische Kontrollen zu umgehen ${ }^{23}$. Auf diese Überlegungen, die nicht nur auf die Frage der Finanzierung, sondern auch auf die Profitabilität verweisen, werden wir zu einem späteren Zeitpunkt zurückkommen.

16 Ibid., S. 199.

17 Dao Thê Tuân, „Les transformations rurales récentes au Vietnam“, Cahiers d'études et de recherches francophones - Agricultures, Bd. 6, Nr. 5, September-Oktober 1997, S. 13-18.

18 Dao Thê Anh, Les ménages agricoles du delta du fleuve Rouge face aux réformes économiques au Vietnam (1988-1997), mémoire de diplôme d'études approfondies, Ecole nationale supérieure agronomique de Montpellier, Université Montpellier I, 1998, S. 88. Lê Duc Thinh, Hoang Khanh Phuong und Dao Thê Anh, „Production et commercialisation des cultures sèches d'hiver“, in Institut national des sciences agronomiques du Vietnam (INSA) und Groupe de recherches et d'échanges technologiques (GRET), Agriculture familiale et gestion des ressources du milieu dans le bassin du fleuve Rouge, Hanoi, Maison d'Edition de l'Agriculture, 1999, S. 76.

19 N. Perrin, ,L'action de l'Etat dans le secteur agricole au Vietnam: situation et problèmes, le crédit rural", in F. Geay und Dâo Thê Tuân, Appui à l'organisation de la production agricole dans le Nord du Vietnam, Hanoi, Maison d'Edition de l'Agriculture, 2000, S. 247.

20 V. Sapin, ,Eléments de réflexion sur le crédit rural au Nord-Vietnam“, in Institut national des sciences agronomiques du Vietnam (INSA) und Groupe de recherches et d'échanges technologiques (GRET), op. cit., S. 132.

21 Hoang Kim Giao und Hoang Vu Cuong, op. cit., S. 153.

22 Tran Thi Quê und Hong Phan, „Financial Mini-Market in Rural Vietnam“, Viet Nam Economic Review, Nr. 5 (50), 1998, S. 37.

23 R. Abrami und N. Henaff, ,The City and the Countryside: Economy, State and Socialist Legacies in the Vietnamese Labour Market“, in M. Beresford und Tran Ngoc Angie (eds.), op. cit., S. 102. 


\section{Erklärungsansätze für das „Agrarwunder"}

Bei der Analyse des Wirtschaftsaufschwungs auf dem Land dreht sich die Debatte hauptsächlich um die Bedeutung der institutionellen (d.h. die Dekollektivierung) und makroökonomischen (Verbesserung der Handelsbedingungen für die Produzenten) Faktoren ${ }^{24}$. In einer ersten Phase wurden die steigenden Landwirtschaftserträge primär auf die Wiederherstellung der freien Wahl in Bezug auf Kulturen und Produktionstechniken zurückgeführt ${ }^{25}$. Auch die Initiativfreiheit galt als entscheidend für den Handel ${ }^{26}$. Andere Autoren dagegen relativierten die Bedeutung der institutionellen Reformen und machten geltend, zwischen der Auflösung der Kooperativen und der Landwirtschaftsproduktion bestünde kein signifikanter Zusammenhang. Zudem habe der Aufschwung in der Agrarwirtschaft in einigen Fällen bereits begonnen, bevor die Familienwirtschaft offiziell rehabilitiert worden $\mathrm{war}^{27}$. Dieser Argumentation liegt die Auffassung zugrunde, wonach nicht die Organisation der Arbeit im Kollektiv das eigentliche Problem sei, sondern vielmehr die Bürokratisierung der Leitung der Kooperativen und die Veruntreuung durch die Verantwortlichen ${ }^{28}$. Der Hauptgrund für den Produktionsaufschwung sei somit hauptsächlich in der makroökonomischen Politik und in den Reformen der Preis- und der Steuersysteme zu suchen, die zu einem Anstieg der landwirtschaftlichen Einkommen geführt und auf diese Weise einen Anreiz für die Bauern geschaffen hätten, sich mehr anzustrengen ${ }^{29}$. Generell werden die öffentliche Politik zur Stützung der Landwirtschaft und namentlich die Bemühungen in den Bereichen Verkehrsinfrastruktur, Bewässerung und Technologie als entscheidend erachtet ${ }^{30}$. Ganz abgesehen von der Frage der Verfügbarkeit von Fremdkapital und der Tragfähigkeit des Finanzsystems wird dem

24 T. Sikor, ,Vietnam's Agricultural Miracle: A Preliminary Analysis of Its Causes“, Vietnam's SocioEconomic Development (Hanoi, Institute of Economics, National Centre for Social and Human Sciences), Nr. 9, Frühling 1996, S. 40-56.

25 P. L. Pingali und Vo Tông Xuan, ,Vietnam: Decollectivization and Rice Productivity Growth“, Economic Development and Cultural Change (University of Chicago), Bd. 40, Nr. 4, Juli 1992, S. $697-$ 718. Tran Thi Quê, „Impacts of Institutional Reforms on Agricultural and Rural Development“, Vietnam's Socio-Economic Development (Hanoi, Institute of Economics, National Centre for Social and Human Sciences), Nr. 6, Sommer 1996, S. 25-39.

26 Lê Thanh Nghiep, „Agricultural Development in Vietnam: Issues and Proposals for Reform“, in Mya Than and J. L. H. Tan (eds.), Vietnam's Dilemmas and Options, Singapore, Institute of Southeast Asian Studies, 1991, S. 144-156.

27 B. Tria Kerkvliert und M. Selden, „Agrarian Transformation in China and Vietnam“, The China Journal, Nr. 40, Juli 1998, S. 50.

28 Nguyên Duc Nhuân, „Le district rural vietnamien ou l'Etat en campagne“, in J. Matras-Guin und C. Taillard (dir.), Habitations et habitat d'Asie du Sud-Est continentale. Pratiques et représentations de l'espace, Paris, L'Harmattan, 1992, S. 343-376. Lam Thanh Liêm, Viêt-Nam. Agriculture 19881993. Problèmes et perspectives, Paris, Duong Moi ; La Voie Nouvelle, 1993, 84 S.

29 G. Irvin, „Vietnam: Assessing the Achievements of Doi Moi“, The Journal of Development Studies, Bd. 31, Nr. 5, Juni 1995, S. 725-750. Le Dang Doanh und A. McCarty, „Economic Reform in Vietnam: Achievements and Prospects“, in S. F. Naya und L. H. Tan (eds.), Asian Transitional Economies: Challenges and Prospects for Reform and Transformation, Singapore, Institute of Southeast Asian Studies, 1995, S. 99-153.

30 Vo Tông Xuan, „Sustaining Diversification in Rice Areas“, Vietnam's Socio-Economic Development (Hanoi, Institute of Economics, National Centre for Social and Human Sciences), Nr. 13, März 1998, S. 44-55. 
Kreditangebot - obwohl sich dieses im Anschluss an die Krise der 1980er-Jahre substanziell erhöhte - nur eine relativ geringe Bedeutung zugesprochen.

\section{Versorgung der ländlichen Wirtschaft mit Fremdkapital}

Die Ausweitung des Kreditangebots (siehe unten Schema 2) wurde in erster Linie durch die Sparguthaben möglich, die in der Ära der Planwirtschaft angesichts des generellen Mangels an Konsumgütern und privaten Entfaltungsmöglichkeiten angehäuft worden waren ${ }^{31}$. Sobald die Anerkennung der Privatwirtschaft gesichert schien, verwendeten die Haushalte ihre Sparguthaben (sofern sie welche besassen) zur Finanzierung ihrer eigenen Produktionstätigkeit oder liehen das Geld an andere Familien aus. Vor allem nach dem Zusammenbruch der Kreditgenossenschaften stellten Darlehen an andere Familienbetriebe die interessanteste Anlagemöglichkeit dar. Mitte der 1990er-Jahre bot die Vietnamesische Agrarbank (BAV) ${ }^{32}$ für Einlagen über mindestens ein Jahr eine Verzinsung von 0,8\%, während Darlehen an Private (d.h. an andere Familien) 3 bis $4 \%$ Zinsen pro Monat einbrachten. Das Zinsgefälle war so stark, dass die BAVFiliale in Châu Giang ${ }^{33} 1998$ dazu überging, die Jahreszinsen für auf ein Jahr gebundene Einlagen im Voraus auszuzahlen, in der Hoffnung, so mehr Sparkunden zu gewinnen. Dem Bankensystem gelang es offensichtlich nur mit grosser Mühe, in ländlichen Gebieten Spareinlagen zu mobilisieren - nicht nur wegen der tiefen Verzinsung, sondern auch wegen des mangelnden Vertrauens der Bevölkerung in die Institution Bank. Die Ergebnisse der Feldforschung in Châu Giang stimmen mit jenen von McCarty weitgehend überein ${ }^{34}$.

So wurden die Familienersparnisse hauptsächlich in Form von Darlehen an Familien aus demselben Dorf oder derselben Gemeinde in die lokale Wirtschaft investiert, in einem Kontext also, in dem der gesellschaftliche Druck auf säumige Gläubiger am stärksten ist. Verschiedene Untersuchungen über die Entwicklung des Kreditwesens in ländlichen Gegenden stellen mit Befriedigung fest, dass der Anteil der privaten Darlehen am gesamten Kreditvolumen aufgrund ihrer zuweilen an Wucher erinnernden hohen Verzinsung rückläufig ist. Dennoch darf nicht vergessen werden, dass diese Darlehen insofern entscheidend waren, als sie für die Haushalte die erste Möglichkeit überhaupt darstellten, an Fremdkapital zu kommen. Ausserdem finden sich unter den Familien, die private Darlehen aufnehmen, gerade auch jene, die am wenigsten Chancen auf einen Kredit der BAV haben: Im Unterschied zu einem Kredit der BAV nämlich, der punktuell aufgenommen werden kann, lässt sich mit einer Vielzahl von privaten Darlehen längerfristig ein Verschuldungsgrad aufrechterhalten, der

31 Der Umfang dieser Sparguthaben ist nicht einmal schätzungsweise bekannt. Hoang Kim Giao spricht vom Dreifachen des vietnamesischen Staatshaushaltes zu Beginn der 1990er-Jahre (zitiert in A. Woodside, „The Struggle to Rethink the Vietnamese State“, in T. Brook und Hy Van Luong (eds.), Culture and Economy: The Shaping of Capitalism in Eastern Asia, Chicago, University of Michigan Press, 1997, S. 63.

32 Die Agrarentwicklungsbank (VBARD) war 1991 in Vietnamesische Agrarbank (BAV) umbenannt worden.

33 Der Landkreis Châu Giang war 1999 noch in die beiden Kreise Khoai Châu und Van Giang aufgeteilt.

34 A. McCarty, Microfinance in Vietnam: A Survey of Schemes and Issues, State Bank of Vietnam; UK Department for International Development (DFID), April 2001, S. 20, <http://ideas.repec.org/p/wpa/ wuwpfi/0110001.html> 
zwar das Einkommen belastet, aber gleichzeitig eine Erhöhung oder zumindest eine Beibehaltung des Tätigkeitsvolumens ermöglicht ${ }^{35}$.

Abgesehen von diesen lokalen Kapitalquellen profitierten die ländlichen Regionen von einem Geldzustrom, der durch die aufblühenden Handelsbeziehungen nach aussen, durch die von den Händlern in den Städten auf Kredit gelieferten Waren und durch die Lieferantenkredite der Industrieunternehmen gespeist wurde. Für Händler, die ein eigenes Geschäft eröffneten, wurde es sehr bald zur Regel, einen Teil der Warenlieferungen erst später zu bezahlen, denn unter den Grossverteilern und Herstellern von landwirtschaftlichen Hilfsstoffen, Baumaterialien und Getränken usw. herrschte ein lebhafter Wettbewerb. Somit waren auch die kleinen Ladenbesitzer in der Lage, den Dorfbewohnern landwirtschaftliche Hilfsstoffe, alltägliche Konsumgüter, Baumaterialien und Einrichtungsgegenstände zunehmend auf Kredit zu verkaufen.

Die Landbevölkerung begann auch ausserhalb der Dörfer um Kredit anzusuchen. Ein Beispiel dafür sind die Agrargrosshändler, die ganze Ernten aufkauften, um sie auf den Märkten im Süden des Landes oder in China zu verkaufen: Sie liehen sich das Geld für ihre Einkäufe bei Gruppen von Kreditgebern (nhom doi chuyên tien) in Hanoi aus. Ähnlich verhält es sich mit den fahrenden Händlern: Sie liessen sich von Händlern in Hanoi Einkäufe von Waren bevorschussen, die sie in andere nordvietnamesische Städte transportierten und dort verkauften. Auf dem Rückweg beförderten sie andere Erzeugnisse aus diesen Städten und insbesondere aus China, die für die Händler in Hanoi bestimmt waren, die ihnen den Vorschuss gewährt hatten. Für die Familienbetriebe, die den Kauf solcher Warenmengen nicht aus eigener Kraft hätten finanzieren können, wäre eine solche Geschäftstätigkeit ohne das System der Vorschüsse nicht in Frage gekommen. Als die Polizeikontrollen auf den Handelsrouten verschärft wurden, um gegen illegale Einfuhren aus China vorzugehen, verzeichnete die Branche einen markanten Einbruch: Die Grosshändler in Hanoi zeigten wenig Bereitschaft, Vorschüsse für Waren zu gewähren, die vielleicht gar nie ankommen würden, denn im Falle einer Beschlagnahmung wäre der Transporteur nicht in der Lage, den Vorschuss zurückzuzahlen.

Dieses zunehmende Kreditangebot seitens privater Wirtschaftsakteure wurde ergänzt durch jenes des ebenfalls expandierenden Bankensektors. Die in bedeutendem Umfang vom Staat finanzierte Vietnamesische Agrarbank (BAV) erhielt den Auftrag, Kredite künftig direkt an die Familien zu vergeben ${ }^{36}$. In der Folge verzeichnete das Kreditgeschäft mit den Familienbetrieben einen rapiden Aufschwung. 1995 stellten die Darlehen an Familien knapp 80\% des Kreditvolumens. Auf Initiative der Vietnamesischen Zentralbank wurden die Kreditgenossenschaften, die die Krise der ausgehenden 1980er-Jahre überlebt hatten, 1994 in Volkskreditfonds (Quy Tin Dung) umgewandelt. Allerdings war zu Beginn das Misstrauen der Bevölkerung gegenüber diesen Fonds gross: Nur wenige Familien mit eigenen Ersparnissen liessen sich davon überzeugen, die für einen Beitritt erforderlichen Anteile zu erwerben, zumal Spareinlagen verglichen mit einem privat gewährten Darlehen kaum Zinsen einbrachten. Nicht zuletzt wurden diese Fonds durch Vertreter der lokalen Behörden und der oberen Gesellschaftsschicht geführt, was dem Vertrauen nicht unbedingt förderlich war.

35 C. Ménard, op. cit., S. 303 ff.

36 A. McCarty, op. cit., S. 9 und 11. 


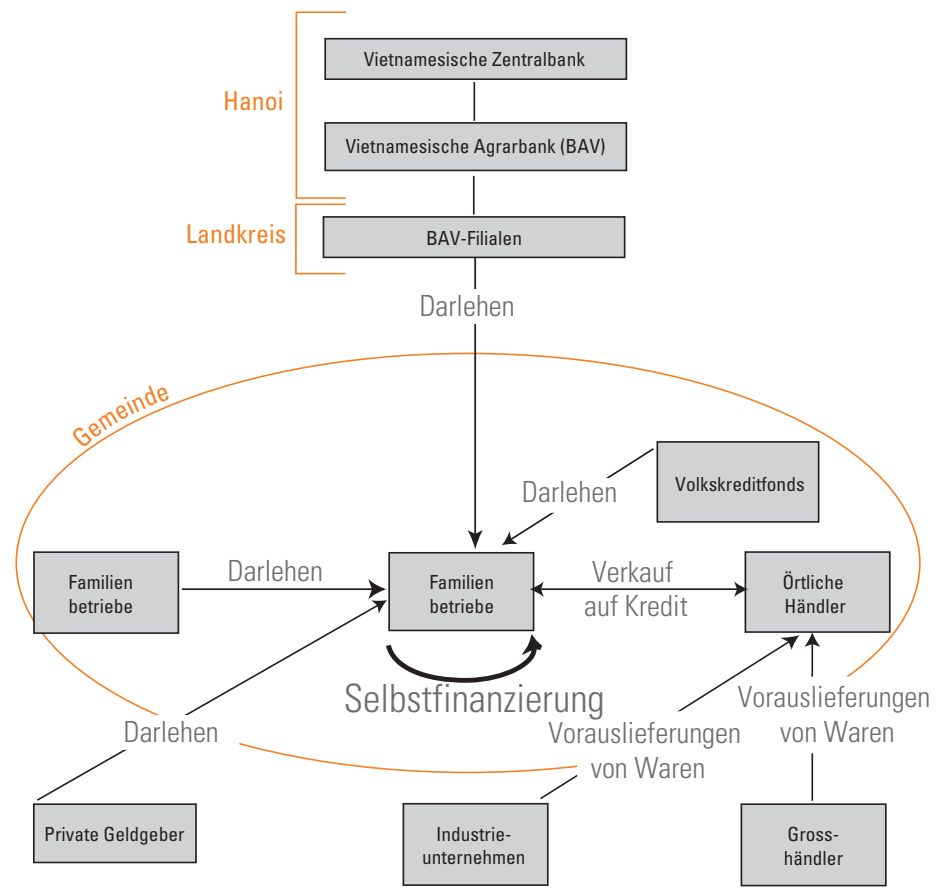

Die Zunahme des Kreditangebots in den 1990er-Jahren war beträchtlich. Die Familienwirtschaft verzeichnete dank einer regen Abfolge von Krediten einen Aufschwung: Bevor ein Kredit fällig wurde, wurde bereits der nächste aufgenommen. Auf diese Weise beschleunigte sich der Geldumlauf, und die aufgrund der landwirtschaftlichen Produktionszyklen saisonalen Schwankungen der Kapitalverfügbarkeit konnten überwunden werden. Nachdem in einer ersten Phase die Leistungsfähigkeit der Landwirtschaft vor allem durch einen zunehmenden Einsatz von Arbeitskraft erhöht wurde, ermöglichte nun die Ausweitung des Kreditangebots die Umstellung auf kapitalintensivere Anbau- und Zuchtsysteme. Auch die Verbesserung der Wohnverhältnisse, der Bau und die Einrichtung neuer Häuser und der Erwerb von Unterhaltungselektronikprodukten wären ohne Fremdkapital nicht möglich gewesen: Wie auch bei den Investitionen in die Produktionsmittel hätten Einkommen und Vermögen der Haushalte allein dazu nicht ausgereicht. Für die ärmsten Haushalte ist die Verweigerung eines Kredits oder des Einkaufens auf Kredit in den örtlichen Geschäften eine gravierende Form der Exklusion, denn sie können ihren Familienbetrieb nur dank hoher Verschuldung über Wasser halten.

Der Umfang der Erhöhung des Kreditangebots und vor allem die Kreditgewohnheiten lassen sich nur schwer beziffern: Gewissen Schätzungen zufolge nehmen rund drei Viertel aller Familienbetriebe Kredite in irgendeiner Form in Anspruch ${ }^{37}$.

37 Vietnamese Consultative Group, Vietnam Development Report 2004: Poverty, Joint Donor Report to the Vietnamese Consultative Group Meeting (2003), Hanoi, Dezember 2003, S. 43. Der Bericht präzisiert, dass in dieser Zahl die Haushalte berücksichtigt sind, die in den Genuss von Krediten des Programms zur Hunger- und Armutsbekämpfung gekommen sind. 
Die Aussagekraft aggregierter Schätzungen ist begrenzt, denn Daten sind nur für das formelle (hauptsächlich über die Agrarbank) und das semiformelle Kreditwesen (Kreditprogramme, die gemeinsam mit internationalen Organisationen und ausländischen Entwicklungshilfeagenturen lanciert wurden) verfügbar: Man weiss zwar, dass sich deren Volumen erhöht hat und dass sie die privaten Darlehen teilweise ersetzt haben, der genaue Umfang ist jedoch unbekannt ${ }^{38}$. Die Daten geben zwar Auskunft über die Zahl der Kredite; in Bezug auf die Beträge sind sie jedoch weniger aufschlussreich, sodass über die Höhe der aufgenommenen Kredite und die Kapitalbewegungen zur Finanzierung der Familienbetriebe nur spekuliert werden kann. Vor allem zum informellen Kreditwesen sind praktisch keine quantitativen Untersuchungen verfügbar. Sein Anteil am gesamten Kreditvolumen im Jahr 2002 wird auf 35\% geschätzt ${ }^{39}$, und im selben Jahr dürften rund $77 \%$ aller Haushalte einen solchen Kredit in Anspruch genommen haben ${ }^{40}$. Die Kreditgewohnheiten und insbesondere die Teilnahme an Kreditvereinigungen oder Tontinen (hui ho, ho phuong) lassen sich nur äusserst schwer beziffern. Auch über den Umfang der von Grosshändlern und Industrieunternehmen auf Vorschuss gelieferten Waren sind keine Angaben verfügbar. Dabei war gerade diese Komponente des Kreditwesens für den Aufschwung der Familienwirtschaft entscheidend, denn auf der Ebene der Familienbetriebe entfalten diese Vorschüsse, die von der Einbindung in die Produktionskette zeugen, eine beträchtliche Hebelwirkung.

Von der Entwicklung des Kreditwesens haben alle Kategorien von Familienbetrieben profitiert, wenn auch in unterschiedlichem Umfang und mit unterschiedlicher Wirkung. Diese Unterschiede gilt es bei den Überlegungen zu der dominierenden und nur selten hinterfragten These vom Mangel an Fremdkapital zu berïcksichtigen.

\section{Beziehungsnetzwerke und Profit}

Die Finanzierungsfrage lässt sich indessen nicht nur unter dem Blickwinkel des Mangels an Fremdkapital betrachten. Dies zeigt die Entwicklung der Familienunternehmen, die stattgefunden hat, ohne dass im Vorfeld rasch und in grossem Umfang Kapital angehäuft wurde. Zugleich lässt sich feststellen, dass ein ebenfalls wachsender Teil der Familien trotz des erweiterten Angebots auf Kredite verzichtet.

\section{Beziehungen vor Kredit}

Ein wesentliches Merkmal der wirtschaftlichen Entwicklung in den ländlichen Regionen Vietnams ist die Entstehung von Familienunternehmen, die im Handel, im Dienstleistungssektor, im Kleingewerbe und in der Verarbeitung von Agrarerzeugnissen tätig sind. Die Umsatzzahlen und die Ausstattung dieser Betriebe mit Ressourcen (Maschinen, Lagerbeständen, Immobilien, Fahrzeugen) lassen sich

38 A.-C. Creusot et al., op. cit. M. Barslund und F. Tarp, Rural Credit in Vietnam, Discussion Paper, Nr. 06-03, Department of Economics, Copenhagen, University of Copenhagen, 2006.

39 M. Barslund und F. Tarp, op. cit.

40 Pham Bao Duong, Review of Rural Finance in Vietnam, Hanoi, Ministry of Planning and Investment, Dezember 2003, zitiert in M. Leflart, op. cit., S. 8. 
heute in keiner Weise mit jenen der landwirtschaftlichen Familienbetriebe vergleichen. Verwundern mag indessen der geringe Kapitaleinsatz, mit dem diese Unternehmen gegründet und später weiterentwickelt wurden. Nicht selten begann die Unternehmenstätigkeit mit dem gelegentlichen Verkauf oder der Herstellung einer kleinen Auswahl an Produkten oder mit sporadisch erbrachten Dienstleistungen. In dieser Anfangsphase waren weder Eigenkapital noch bedeutende Fremdmittel erforderlich: Das Geschäftsvermögen beschränkte sich auf ein Minimum, und die Produkte wurden häufig von Händlern auf Vorschuss geliefert, die sich zusätzliche Absatzkanäle erschliessen wollten. Die Entwicklung dieser Unternehmen erfolgte schrittweise: Genügte zunächst ein Tisch direkt vor dem Haus als Auslage, so wurde die Palette der angebotenen Waren und Dienstleistungen nach und nach erweitert. Es folgten eine Ausdehnung des Produktionsvolumens, in manchen Fällen ein Einstieg in den Grosshandel und schliesslich die Einstellung einiger Angestellter, die die Arbeitskraft der Familie ergänzten. Diese Entwicklung dauerte drei, fünf oder zehn Jahre, wenn nicht länger. Gewiss waren auch Investitionen erforderlich, die teils über Kredite, aber auch über reinvestierte Gewinne, über Vorauslieferungen von Grosshändlern und über Lieferantenkredite der Industrieunternehmen finanziert wurden. Zahlreiche Handwerker konnten ihren Betrieb dank Verträgen mit Möbelherstellern ausbauen, die ihnen das Holz lieferten, aus dem sie Möbelteile fertigten. Das Fremdkapital war zweifellos eine von mehreren verschiedenen Voraussetzungen für die Entwicklung dieser Unternehmen, aber sicherlich nicht so entscheidend, wie in zahlreichen Untersuchungen über die Grenzen der Entwicklung der Familienwirtschaft und der Kleinunternehmen behauptet wird.

Der Aufschwung dieser Unternehmen deutet darauf hin, dass bei der Ausdehnung der Tätigkeit über den dörflichen Rahmen hinaus Beziehungen zu Partnern wichtiger sind als die verfügbaren Finanzmittel. Ein treffendes Beispiel hierfür sind die Unternehmen, die Agrarerzeugnisse verarbeiten. Ausschlaggebend für den Entschluss dieser Familien, ihr Unternehmen zu gründen, waren ihre Bekanntschaften mit Akteuren, die selbst im Bereich der Weiterverarbeitung von Agrarprodukten tätig waren, sowie die Beziehungen, die mit und über Vertrauenspersonen geknüpft wurden. Im Falle der Verarbeitung und des Vertriebs von Agrarerzeugnissen sind dies im nachgelagerten Bereich Käufer, die Bestellungen aus Saigon oder aus China vermitteln, sowie Transporteure, die die Beförderung und Lieferung der Produkte ohne Umweg und ohne Unterschlagung sicherstellen und dafür sorgen, dass die bestellten Waren bezahlt werden. Im vorgelagerten Bereich sind es die Einkäufer von Frischprodukten, die für eine pünktliche Lieferung und eine einwandfreie Qualität der zu verarbeitenden Güter bürgen, und die Bereitschaft der Produzenten, ihre Ernte auf Kredit zu verkaufen. Entscheidend sind die Zuverlässigkeit und Tragfähigkeit dieser Partnerschaften sowie die Gewährleistung, dass keine Geschäfte unter der Hand gemacht und keine Waren gestohlen werden. Die Entstehung dieser Partnerschaften ist eng mit dem beruflichen Werdegang der heutigen Unternehmensführer verknüpft: Beziehungen kamen vorzugsweise über oder mit ehemaligen Kollegen, Schulkameraden oder Familienangehörigen sowie mit ehemaligen Einwohnern derselben Gemeinde zustande. Bei den heutigen Firmeneigentümern handelt es sich um ehemalige Kader und Angestellte der Staatsbetriebe und Kooperativen, die nach dem Übergang zur Marktwirtschaft als Selbstständigerwerbende die Tätigkeit fortsetzten, die sie früher als Angestellte 
ausübten - in manchen Fällen bereits damals unerlaubt auf eigene Rechnung und zum Nachteil der Kooperative oder des Staatsbetriebs. Diese ehemaligen Betriebsleiter, Kader oder auch nur einfachen Angestellten oder Arbeiter wussten nicht nur ausgezeichnet über Produkte, Fertigungstechniken, Versorgungsmöglichkeiten und Absatzmärkte Bescheid, sondern sie kannten auch die Schlüsselakteure der Staatsbetriebe und Kooperativen und waren damit bestens positioniert, um an der Neuorganisation der Wirtschaft teilzuhaben.

Ihren Erfolg verdanken diese Unternehmen dem sozialen Kapital und dem Beziehungsnetz ihrer Eigentümer, aber auch der Qualität der Partnerschaften, die die Entwicklung des Unternehmens und insbesondere die Ausdehnung der Unternehmenstätigkeit auf weiter entfernte Regionen erst möglich machten. Die Kenntnis der Vertriebsnetze und -kanäle ist ein Garant für den unternehmerischen Erfolg und zudem die beste Sicherheit, die die Unternehmen einem Geldgeber bieten können. Der Auf- und Ausbau der Unternehmen war natürlich begleitet von kapazitätssteigernden Investitionen (Lagerräume, Fahrzeuge). Diese Investitionen wurden indessen erst getätigt, als die Unternehmer überzeugt waren, die gesteigerten Produktionsmengen auch tatsächlich absetzen zu können. Die Akkumulation von Kapital war keine Voraussetzung für die Aufnahme der Unternehmenstätigkeit, sondern sie erfolgte erst in einem späteren Stadium der Entwicklung. Die heutigen Umsatzzahlen dieser Unternehmen sind natürlich auch das Ergebnis des investierten Kapitals, aber ihren unternehmerischen Erfolg verdanken sie eher der Qualität ihrer Partnerschaften. Auch heute zeigt sich, dass sich die Weiterentwicklung dieser Unternehmen nicht so sehr an der Verfügbarkeit von Finanzmitteln als an der Erschliessung neuer Absatzmöglichkeiten entscheidet. Diese Unternehmen haben nicht die geringsten Schwierigkeiten, bei der Vietnamesischen Agrarbank (BAV) oder bei einem Volkskreditfonds ein Darlehen zu erhalten. Aber wie verhält es sich mit den einfachen Familienbetrieben?

\section{Mangel an Profit}

Der Begriff „einfache Familienbetriebe“ verweist auf die Tatsache, dass diesen Betrieben wirtschaftliche und technische Grenzen gesetzt sind, die durch die Anbau- und Zuchtsysteme vorgegeben sind. Das Kreditverhalten dieser Betriebe macht deutlich, dass das Fremdkapital hier keine Hebelwirkung entfalten kann. Dieser Sachverhalt wird häufig als Bestätigung für die These des mangelnden Kreditangebots ausgelegt.

Der Kontext präsentiert sich wie folgt: Das Angebot an Finanzierungsmöglichkeiten ist grösser geworden, die Zinsen sind gesunken, und ein wachsender Anteil der bäuerlichen Familienbetriebe ist im Besitz einer Urkunde, die ihnen das Nutzungsrecht für ihr Land bescheinigt und so den Zugang zu Krediten erleichtern soll. Aber das grössere Angebot ist - zumindest was das formelle Kreditwesen betrifft - den finanziell besser gestellten Haushalten vorbehalten, denn die Volkskreditfonds gehen bei der Kreditvergabe nach ähnlichen Grundsätzen vor wie die BAV. Zudem ist der Finanzbedarf der Haushalte ebenfalls gestiegen. Die Betriebsausgaben haben sich erhöht: Neue Betriebe müssen eine Parzelle erwerben, sofern sie keine geerbt haben. Die Bodenpreise sind explodiert, und zwar sowohl für Grundstücke, die von anderen Betrieben gepachtet 
werden, als auch für solche, die öffentlich zugeteilt werden. Grössere Ackerflächen müssen heute zum Zeitpunkt ihrer Zuteilung in bar bezahlt werden. Dazu kommen produktionsbezogene Auslagen: Gewerbsmässig angebaute Kulturen verbrauchen mehr Hilfsstoffe. Zuchtbetriebe müssen Futtermittel kaufen und Ställe bauen. Darüber hinaus müssen die Betriebe zusätzliche Abgaben leisten, die von den Gemeinden zum Zwecke der Modernisierung erhoben werden, etwa für den Bau von Strassen, von neuen Klassenzimmern oder gar von ganzen Schulen. Bei den Familienbudgets schlagen insbesondere die Ausgaben für Bildung und Gesundheit zu Buche, denn diese sind neu zahlungspflichtig. Auch die materiellen Ansprüche und die sozialen Normen, die sich im Zuge des vietnamesischen Wirtschaftswachstums erhöhen, verlangen nach zusätzlichen Ausgaben zur Sicherung der gesellschaftlichen Anerkennung (etwa bei Hochzeitsfeiern oder anderen Familienanlässen) oder des sozialen Aufstiegs (bessere Ausbildung für die Kinder).

Auch die Unwägbarkeiten haben sich erhöht: Der zunehmende Anteil der zum Verkauf bestimmten Produktion sorgt nicht nur für höhere Einnahmen, sondern ist angesichts des heutigen Entwicklungsstandes und der gegenwärtigen Marktregulierung auch mit grösseren Risiken verbunden ${ }^{41}$. Die Preise für Agrarprodukte sind volatiler - der Zerfall des Kaffeepreises in den Jahren 1999 und 2000 ist ein schlagendes Beispiel dafür -, und die vorübergehende Sättigung der Märkte bewirkt schmerzhafte Einkommensverluste, sofern überhaupt ein Abnehmer gefunden werden kann. Angesichts dieser Risiken - und der Höhe des zu erwartenden Einkommens - verfolgen die Produzenten in Bezug auf die Auswahl der Kulturen und Sorten und auf das technische Vorgehen eine hinlänglich bekannte Strategie: Sie entscheiden sich für Produktionssysteme, die das Potenzial ihrer Anbauflächen nicht vollständig ausschöpfen.

Im Reisanbau beispielsweise liegen die Erträge seit Mitte der 1990er-Jahre relativ konstant bei 5,5 Tonnen Paddy-Reis pro Hektare. Nur wenige Produzenten bauten aus China importierte Hybridsorten an, deren Ertrag bei über 6 Tonnen pro Hektare liegt. Das ungenügende Angebot von Saatgut ist nur einer der Gründe dafür: Aus der Sicht der Bauern sind diese Sorten mit zahlreichen Nachteilen behaftet. Sie werden von den vietnamesischen Verbrauchern aufgrund ihres Geschmacks verschmäht und verkaufen sich somit schlechter als einheimische Sorten. Ausserdem könnte nur im Frühlings- und Sommerzyklus ein Mehrertrag erzielt werden, denn im zweiten Anbauzyklus von Juni bis Oktober fällt mehr Regen, und die Hybridsorten sind anfälliger auf Fäulnis. Ein weiterer Grund ist, dass das Saatgut teurer ist als jenes für einheimische Sorten, und da es sich um Hybride handelt, muss für jeden Anbauzyklus neues Saatgut beschafft werden. Nicht zuletzt benötigen diese Sorten mehr Dünger und Pflanzenschutzmittel als einheimische Sorten. Unter dem Strich zeigt sich sehr schnell, dass mit sogenannt „optimierten“ Sorten nicht unbedingt ein höheres Einkommen erzielt wird. Mit der Schweinezucht verhält es sich ähnlich ${ }^{42}$. Das

41 P. Bergeret und Pham Hoang Ha, „Dynamiques comparées de trois filières dans le delta du fleuve Rouge au Nord-Vietnam“, Gemeinschaftsausgabe von Cahiers agriculture, Bd. 6, Nr. 5, und Agriculture et Développement, Nr. 15, 1997, S. 19-25.

42 K. Le Goulven, Dispositifs institutionnels et intégration des marchés. La commercialisation du porc au Vietnam, thèse de doctorat, Montpellier, Ecole nationale supérieure agronomique de Montpellier, Juli 2000, 403 S. 
Problem liegt eindeutig beim Profit: Er ist zu tief, um Produzenten mit den nötigen Mitteln zu veranlassen, in den Reisanbau zu investieren, und zwar ist er so gering, dass mit Lohnarbeit mehr verdient werden kann.

Daran ändert auch die Verfügbarkeit von Fremdkapital nichts. Das haben Kalkulationen über den Return on Investment für die Pacht von Land für den Reisanbau oder die Schweinezucht auf Kredit eindeutig gezeigt ${ }^{43}$. Bereits vor etwa zehn Jahren zogen es manche Haushalte vor, die ihnen zugewiesenen Reisfelder zu verpachten, anstatt sie selber zu bewirtschaften ${ }^{44}$. Es war einträglicher, sich als Kleinhändler oder Dienstleister zu betätigen oder seine Arbeitskraft zu vermieten. Für Investitionen in die Landwirtschaft entschieden sich nur Familien, die keine anderen Möglichkeiten hatten. Dies bestätigen Umfragen, die im Herbst 2006 in Châu Giang durchgeführt worden sind. Diese Haushalte bemühen sich gar nicht erst darum, weitere Anbauflächen zugewiesen zu erhalten. Zahlreiche Bewohnerinnen und Bewohner von Châu Giang pendeln entweder zur Arbeit nach Hanoi oder versuchen, tageweise in einer der Fabriken zu arbeiten, die in den letzten paar Jahren im Kreis entstanden sind. Wenn sie einen Kredit aufnehmen - in der Regel beträchtliche Summen -, dann nur deshalb, um die Teilnahme an einem Programm zu finanzieren, das ihnen in Malaysia, in Taiwan oder in Südkorea zu einer Arbeit verhilft. Dieses Kreditverhalten orientiert sich ausschliesslich an dem erwarteten Einkommen und den damit verbundenen Risiken; das Kreditangebot selbst spielt nur eine untergeordnete Rolle. Angesichts der zu hohen Risiken, die mit ertragreicheren Kulturen verbunden sind, und des zu tiefen Einkommens, das mit der Grundproduktion erzielt werden kann, beschränken sich die Möglichkeiten zahlreicher Familien auf jene landwirtschaftlichen Tätigkeiten, die kein Fremdkapital erfordern, obwohl sich die Kreditkonditionen verbessert haben.

\section{Fazit}

Die institutionellen Reformen im vietnamesischen Landwirtschaftssektor bewirkten, dass die Familienbetriebe ihre Produktionstätigkeit selbst finanzieren mussten. Zusammen mit der rasanten Entwicklung von Anbau- und Zuchtsystemen, die immer grössere Vorschüsse und Investitionen erforderten, mündete dies in einen Mangel an Finanzkapital. Dieser nicht bezifferbare, aber dennoch offenkundige Mangel prägte die ausgehenden 1980er- und die erste Hälfte der 1990er-Jahre. In dieser Übergangsphase, die durch den Zusammenbruch des Systems der Kreditgenossenschaften gekennzeichnet war, fehlte es den Bauern in der Tat an Fremdkapital.

Angesichts des Wandels, den die ländliche Wirtschaft seither durchlaufen hat, müssen die Bedeutung, die dem Kreditwesen beigemessen wird, aber auch die noch immer aktuelle These vom Mangel an Fremdkapital als entscheidendem

43 C. Ménard, op. cit., S. 179-181.

44 Kraft des Grundeigentumsgesetzes von 1993 wurden $85 \%$ aller Reisanbauflächen für eine Dauer von 30 Jahren den einzelnen Familien überlassen. Die verbleibenden $15 \%$ dienen als Reserve für Betriebe, die im Laufe dieser 30 Jahre gegründet würden. In der Zwischenzeit werden diese Anbauflächen per Auslosung für eine Dauer von zwei bis fünf Jahren an einzelne Betriebe zugewiesen. Entschieden früher das Los oder der Gemeindevorstand und die Führung des Volksausschusses der Gemeinde über die Zuweisung, so werden die Reisfelder heute mehrheitlich versteigert. 
Faktor für die Entwicklung der Familienwirtschaft und deren Grenzen revidiert werden. Die wichtige Rolle des Fremdkapitals ist unbestritten: Die markante Ausweitung des Kreditangebots hat zur Entstehung einer kapitalintensiveren Landwirtschaft, zur Diversifizierung der ländlichen Wirtschaft und zur Verbesserung der materiellen Lebensbedingungen der Bevölkerung beigetragen. Indessen hängen die Investitionen und das Kreditvolumen weniger vom Angebot und den Kosten für Fremdkapital ab als von der Rentabilität der Produktionstätigkeit, die damit finanziert werden soll. So verzichten Familienbetriebe trotz dem grösseren Angebot auf Kredite, weil die Erträge aus einer Produktion mit gesichertem Absatz ungenügend sind oder weil sie keine Garantie dafür haben, rentablere Produkte auch tatsächlich verkaufen zu können. Diesen Bauern fehlt es weniger an Fremdkapital denn an Gewinn. Ein Blick auf die Familienunternehmen bestätigt diesen Befund: Ausschlaggebend für ihren wirtschaftlichen Erfolg waren ihre Beziehungsnetzwerke, Bekanntschaften also, die ihnen die Sicherheit bieten konnten, dass sie ihre Produktion auf den nach wie vor wenig entwickelten Märkten absetzen können. Fremdkapital und Investitionen waren keine Vorbedingungen, sondern wurden erst aktuell, als der Absatz gesichert war. Die Rentabilität dieser Unternehmen hängt also stärker von ihrem Beziehungsnetz als vom investierten Kapital ab.

Die Frage, wie die Entwicklung der Familienwirtschaft finanziert werden kann, lässt sich nicht nur unter dem Blickwinkel der Verfügbarkeit und der Kosten von Fremdkapital erörtern. Diese Problematik war aktuell, als es galt, zum Zeitpunkt der Rehabilitation der Familienwirtschaft die Produktion zu erhöhen. Heute jedoch sieht sich die vietnamesische Agrarwirtschaft in erster Linie mit einem Wettbewerbs- und Rentabilitätsproblem konfrontiert, das mit Fremdkapital nicht gelöst werden kann.

\section{Bibliografie}

Abrami, R., und N. Henaff, „The City and the Countryside: Economy, State and Socialist Legacies in the Vietnamese Labour Market“, in M. Beresford und Tran Ngoc Angie (eds.), Reaching for the Dream: Challenges of Sustainable Development in Vietnam, Copenhagen, Nordic Institute of Asian Studies; Singapore, Institute of Southeast Asian Studies, 2004, S. 95-134.

Barslund, M., und F. Tarp, Rural Credit in Vietnam, Discussion Paper, Nr. 06-03, Department of Economics, Copenhagen, University of Copenhagen, 2006.

Bergeret, P., und Pham Hoang Ha, „Dynamiques comparées de trois filières dans le delta du fleuve Rouge au Nord-Vietnam“, Gemeinschaftsausgabe von Cahiers agriculture, Vol. 6, Nr. 5, und Agriculture et Développement, Nr. 15, 1997, S. 19-25.

Bui Van Hung, „Rural Diversification: An Essential Path to Sustainable Development for Vietnam“, in M. Beresford und Tran Ngoc Angie (eds.), Reaching for the Dream: Challenges of Sustainable Development in Vietnam, Copenhagen, Nordic Institute of Asian Studies ; Singapore, Institute of Southeast Asian Studies, 2004, S. 183-215.

Creusot, A.-C., Le paysage du financement du monde rural au Vietnam, Bulletins d'information du mardi (Pôle microfinancement du Centre de coopération internationale en recherche agronomique pour le développement [CIRAD] et du Groupe de recherche et d'échanges technologiques [GRET]), Nr. 16, 14. Mai 2002, <http://microfinancement.cirad.fr/fr/news/bim/Bim-2002/BIM-14-05-02.pdf>.

Creusot, A.-C., Quynh Tran Thi Thanh und Luong Quoc Tuân, „La microfinance a-t-elle encore une place lorsque l'offre de crédit s'étend ?", La microfinance en Asie. Entre traditions et innovations, Pondichéry, Institut français de Pondichéry; Paris, Institut de recherche pour le développement; Karthala, 2005, S. 64-82.

Dao Thê Anh, Les ménages agricoles du delta du fleuve Rouge face aux réformes économiques au Vietnam (1988-1997), mémoire de diplôme d'études approfondies, Ecole nationale supérieure agronomique de Montpellier, Université Montpellier I, 1998, 127 S. 
Dao Thê Tuân, „Les transformations rurales récentes au Vietnam“, Cahiers d'études et de recherches francophones-Agricultures, Vol. 6, Nr. 5, September-Oktober 1997, S. 13-18.

Feuché, C., „Croissance, Etat et marché dans le Vietnam du Dôi Moi“, in S. Dovert und B. de Tréglodé (dir.), Vietnam contemporain, Paris, Les Indes Savantes, 2004, S. 231-263.

Gironde, C., Réhabilitation et transformations de l'économie familiale au Nord-Vietnam. Systèmes d'activités villageois et réseaux de relations dans le delta du fleuve Rouge, thèse de doctorat, Genève, Institut universitaire d'études du développement, 2001, 406 S.

Hoang Kim Giao und Hoang Vu Cuong, ,,Vietnam's Private Economy in the Process of Renovation“, in I. Norlund, C. L. Gates und Vu Cao Dam (eds.), Vietnam in a Changing World, Richmond, UK, Curzon Press, 1995, S. 151-158.

Irvin, G., „Vietnam: Assessing the Achievements of Doi Moi“, The Journal of Development Studies, Vol. 31, Nr. 5, Juni 1995, S. 725-750.

Johnson, A., Microfinance in Vietnam: A Collaborative Study Based upon the Experiences of NGOs, UN Agencies and Bilateral Donors, Hanoi, United Nations Development Programme (UNDP); Consultative Group to Assist the Poor (CGAP), 1996.

Kerkvliert, B. Tria, und M. Selden, „Agrarian Transformation in China and Vietnam“, The China Journal, Nr. 40, Juli 1998, S. 37-48.

Lam Thanh Liêm, Viêt-Nam. Agriculture 1988-1993. Problèmes et perspectives, Paris, Duong Moi; La Voie Nouvelle, 1993, $84 \mathrm{~S}$.

Le Dang Doanh und A. McCarty, „Economic Reform in Vietnam: Achievements and Prospects“, in S. F. Naya und L. H. Tan (eds.), Asian Transitional Economies: Challenges and Prospects for Reform and Transformation, Singapore, Institute of Southeast Asian Studies, 1995, S. 99-153.

Lê Duc Thinh, Hoang Khanh Phuong und Dao Thê Anh, „Production et commercialisation des cultures sèches d'hiver", in Institut national des sciences agronomiques du Vietnam (INSA) et Groupe de recherches et d'échanges technologiques (GRET), Agriculture familiale et gestion des ressources du milieu dans le bassin du fleuve Rouge, Hanoi, Maison d'Edition de l'Agriculture, 1999, S. 65-80.

Leflart, M., Intérêt et apport du micro-crédit, Hanoi, Forum économique et financier franco-vietnamien Le financement du développement, 19.-20. Januar 2005, 14 S.

Le Goulven, K., Dispositifs institutionnels et intégration des marchés. La commercialisation du porc au Vietnam, thèse de doctorat, Montpellier, Ecole nationale supérieure agronomique de Montpellier, Juli 2000, $403 \mathrm{~S}$.

Lê Thanh Nghiep, „Agricultural Development in Vietnam: Issues and Proposals for Reform“, in Mya Than und J. L. H. Tan (eds.), Vietnam's Dilemmas and Options, Singapore, Institute of Southeast Asian Studies, 1991, S. 144-156.

McCarty, A., Microfinance in Vietnam: A Survey of Schemes and Issues, State Bank of Vietnam; UK Department for International Development (DFID), April 2001, <http://ideas.repec.org/p/wpa/ wuwpfi/0110001.html>.

Nguyên Duc Nhuân, „Le district rural vietnamien ou l'Etat en campagne“, in J. Matras-Guin und C. Taillard (dir.), Habitations et habitat d'Asie du Sud-Est continentale. Pratiques et représentations de l'espace, Paris, L'Harmattan, 1992, S. 343-376.

Paquet, E., Le système économique vietnamien 1979-2000. De la réforme à la transformation, thèse de doctorat, Paris, Université Paris VII - Denis Diderot, Juni 2003, 416 S.

Perrin, N., „L'action de 1'Etat dans le secteur agricole au Vietnam: situation et problèmes, le crédit rural“", in F. Geay und Dâo Thê Tuân, Appui à l'organisation de la production agricole dans le Nord du Vietnam, Hanoi, Maison d'Edition de l'Agriculture, 2000, 388 S.

Pham Bao Duong, Review of Rural Finance in Vietnam, Hanoi, Ministry of Planning and Investment, Dezember 2003, zit. in M. Leflart, Intérêt et apport du micro-crédit, Hanoi, Forum économique et financier franco-vietnamien - Le financement du développement, 19.-20. Januar 2005, 14 S.

Pingali, P. L., und Vo Tông Xuan, „Vietnam: Decollectivization and Rice Productivity Growth“, Economic Development and Cultural Change (University of Chicago), Vol. 40, Nr. 4, Juli 1992, S. 697-718.

Poursat, C., „Le crédit aux paysans“, in Association d'amitié franco-vietnamienne (dir.), L'agriculture et la paysannerie vietnamiennes, Paris, L’Harmattan, 2000, S. 65-74.

Ronnas, P., und B. Ramamurthy, Entrepreneurship in Vietnam: Transformation and Dynamics, Copenhagen, Nordic Institute of Asian Studies; Singapore, Institute of Southeast Asian Studies, 2001, 354 S.

Sapin, V., „Eléments de réflexion sur le crédit rural au Nord-Vietnam“, in Institut national des sciences agronomiques du Vietnam (INSA) et Groupe de recherches et d'échanges technologiques (GRET), Agriculture familiale et gestion des ressources du milieu dans le bassin du fleuve Rouge, Hanoi, Maison d'Edition de 1'Agriculture, 1999, S. 127-144. 
Sikor, T., „Vietnam's Agricultural Miracle: A Preliminary Analysis of Its Causes“, Vietnam's SocioEconomic Development (Hanoi, Institute of Economics, National Centre for Social and Human Sciences), Nr. 9, Frühling 1996, S. 40-56.

Tran Thi Quê und Hong Phan, „Financial Mini-Market in Rural Vietnam“, Viet Nam Economic Review, Nr. 5 (50), 1998, S. 29-38.

Tran Thi Quê, „Impacts of Institutional Reforms on Agricultural and Rural Development“, Vietnam's Socio-Economic Development (Hanoi, Institute of Economics, National Centre for Social and Human Sciences), Nr. 6, Sommer 1996, S. 25-39.

Vietnamese Consultative Group, Vietnam Development Report 2004 : Poverty, Joint Donor Report to the Vietnamese Consultative Group Meeting (2003), Hanoi, Dezember 2003.

Vo Tông Xuan, „Sustaining Diversification in Rice Areas“, Vietnam's Socio-Economic Development (Hanoi, Institute of Economics, National Centre for Social and Human Sciences), Nr. 13, März 1998, S. 44-55.

Wolz, A., The Transformation of Rural Finance Systems in Vietnam, Discussion Paper, Nr. 60, Research Centre for International Agrarian and Economic Development, Heidelberg, Universität Heidelberg, 1997, $29 \mathrm{~S}$.

Woodside, A., „The Struggle to Rethink the Vietnamese State“, in T. Brook und Hy Van Luong (eds.), Culture and Economy: The Shaping of Capitalism in Eastern Asia, Chicago, University of Michigan Press, 1997, S. 61-78. 